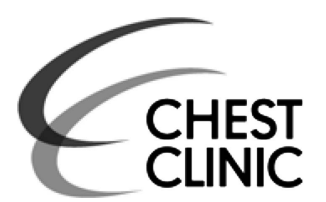
CLHEST

${ }^{1} O x f o r d$ Centre for Respiratory Medicine, Churchill Hospital, Oxford, UK

${ }^{2}$ Department of Radiology, University Hospitals Coventry and Warwickshire NHS Trust, Coventry, UK

${ }^{3}$ Academic Respiratory Unit, School of Clinical Sciences, University of Bristol, Bristol, UK ${ }^{4}$ Division of Pulmonary and Critical Care Medicine, Johns Hopkins Hospital, Baltimore, USA

${ }^{5}$ Department of Radiology, Churchill Hospital, Oxford, UK

\section{Correspondence to} Dr loannis Psallidas, Oxford Centre for Respiratory Medicine, Churchill Hospital, Oxford University Hospitals Trust, Old Road, Oxford OX3 7LE, UK: ioannis.psallidas@ndm.ox.ac uk

Received 6 December 2014 Revised 30 March 2015 Accepted 13 April 2015 Published Online First 1 May 2015

\title{
latrogenic injury to the intercostal artery: aetiology, diagnosis and therapeutic intervention
}

\author{
Ioannis Psallidas, ${ }^{1}$ Emma J Helm, ${ }^{2}$ Nick A Maskell, ${ }^{3}$ Lonny Yarmus, ${ }^{4}$ \\ David J Feller-Kopman, ${ }^{4}$ Fergus V Gleeson, ${ }^{5}$ Najib M Rahman ${ }^{1}$
}

Pleural interventions are commonly performed in both elective and emergency settings. They include simple thoracocentesis, closed pleural biopsy (with or without image guidance), intercostal drain (ICD) insertion, in-dwelling pleural catheter insertion and medical thoracoscopy. Complications of pleural procedures are common but their incidence is often under-recognised. Higher operator experience and the use of image guidance are key factors demonstrated to significantly reduce the frequency of complications. ${ }^{1}$

Injury to the intercostal artery (ICA) is an infrequent but potentially life-threatening complication of all pleural interventions. Pleural haemorrhage is reported to occur in up to $2 \%$ of thoracocenteses, up to $13 \%$ of ICD insertions and up to $4 \%$ of thoracoscopies. $^{2}$ The true incidence of ICA laceration and consequent pleural haemorrhage is likely to be higher due to under-reporting of complications seen in retrospective case series.

The British Thoracic Society has published guidelines for the insertion of ICDs, ${ }^{1}$ aiming to reduce the potential harm of ICD insertion. Although these recommendations are likely to reduce certain complications such as drain insertion into abdominal or thoracic viscera, they do not specifically address the possibility of ICA injury. Proper site selection for pleural interventions is important as this minimises the likelihood of ICA laceration. In a recent large study, Helm et $a l^{3}$ identified that ICA is exposed within the intercostal space in the first $6 \mathrm{~cm}$ lateral to the spine using CT pulmonary angiograph and mapping of the ICA course. The variability of ICA is greater in older people and in more cephalad rib spaces and decreases with lateral distance from the spinous process. Additionally, another important parameter is the management protocol for intrapleural haemorrhage. This should be in place prior to any pleural interventions to avoid life-threatening delays.

We present three cases of iatrogenic ICA injury in different clinical circumstances, from three different institutions in the UK and USA and make recommendations for avoiding and dealing with ICA injury.

\section{CASE 1}

A middle-aged patient was admitted with acute left-sided pleuritic pain and sudden onset dyspnoea. Four months prior to this admission, the patient underwent a left upper lobe wedge resection for a T1 N0 M0 adenocarcinoma of the lung. Medical history also included epilepsy, transient ischaemic attack, hypertension and alcohol abuse. The initial differential diagnosis included pulmonary embolus and therefore treatment dose low molecular weight heparin was given and a CT pulmonary angiogram (CTPA) was arranged. The CTPA did not show any evidence of pulmonary embolus but demonstrated a new small right pleural effusion.

The low molecular weight heparin was stopped for $24 \mathrm{~h}$ and a diagnostic thoracentesis performed using a $21 \mathrm{G}$ needle under ultrasound guidance with successful aspiration of pleural fluid on the second pass. The position chosen for the aspiration was in the ninth intercostal space posteriorly, $8 \mathrm{~cm}$ lateral to the spinal process. Twenty-four hours later, the patient became increasingly short of breath and hypotensive with a drop in haemoglobin. Urgent repeat CT (figure 1) showed highly echogenic material in the pleural fluid and a site of contrast extravasation which was an evidence of fresh haemorrhage. The patient required a blood transfusion and underwent urgent thoracic surgery, where the damaged ICA was identified and ligated. Subsequently, the patient made full recovery apart from minor right-sided pleural thickening evident on the chest radiograph, but this was not associated with any functional deficit.

\section{CASE 2}

A patient with a history of liver transplantation 5 years previously was admitted to the ward for investigation and management of renal failure. During the inpatient stay, the patient developed a large left-sided pleural effusion with associated shortness of breath. An $8 \mathrm{~F}$ pigtail catheter was inserted with no ultrasound guidance on the ward with no apparent immediate complications. However, $1 \mathrm{~h}$ later the patient became increasingly dyspnoeic and was noted to have a large fall in haemoglobin requiring a 6 -unit blood transfusion.

A contrast-enhanced CT scan was performed, which showed a large haemothorax and a jet of contrast originating adjacent to the tip of the chest drain, next to the 10th rib posteriorly. Bleeding from an ICA laceration was suspected and the patient was taken to the interventional radiology suite where embolisation of the ICA was successfully performed with two $3 \mathrm{~mm}$ coils via a $3 \mathrm{~F}$ Progreat catheter (Terumo, Japan).

\section{CASE 3}

A patient with a medical history of COPD, peripheral artery disease and coronary artery disease was 


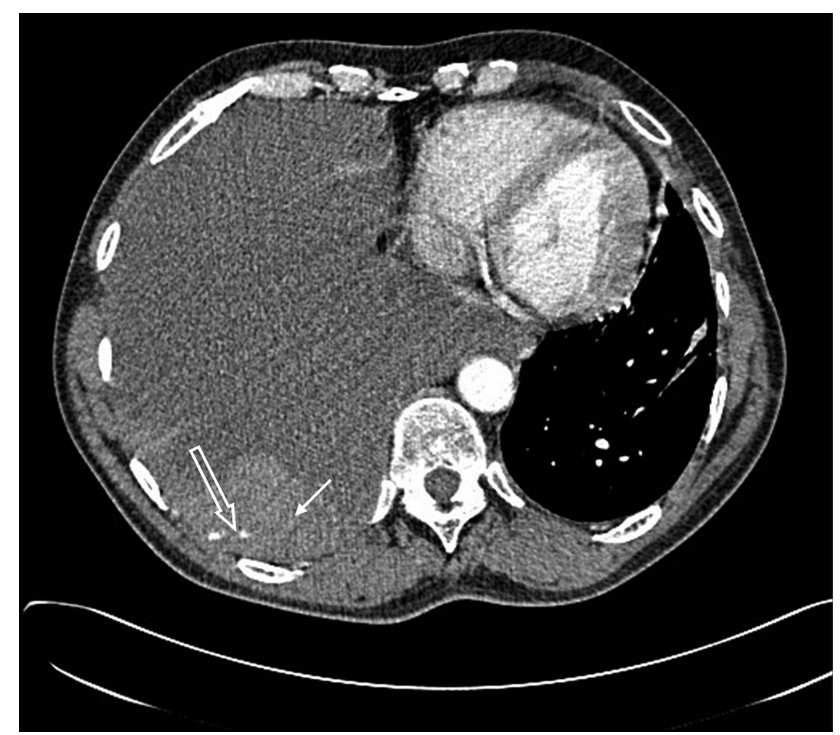

Figure 1 Case 1. CT shows hyperdense blood (closed arrow) and contrast extravasation (open arrow).

admitted with dyspnoea and a left-sided pleural effusion on chest radiograph. A chest ultrasound was performed, which revealed a moderate left-sided hypoechoic effusion. The patient underwent therapeutic thoracentesis using a standard $8 \mathrm{~F}$ catheter-over-the-needle kit, in the ninth intercostal space, $7 \mathrm{~cm}$ lateral to the vertebral spinous process. One thousand millilitres of serous pleural fluid was removed and the procedure was terminated for lack of further fluid. A chest radiograph immediately post procedure showed an interval decrease in the left pleural fluid with no pneumothorax.

Four hours post procedure, the patient developed hypoxia, hypotension and progressive dyspnoea. A repeat chest radiograph showed reaccumulation of the left-sided pleural effusion. Two hours later, another chest film demonstrated that the pleural effusion had enlarged and the patient's haematocrit had dropped from $31 \%$ to $21 \%$. This prompted a chest CT that showed hyperdense left-sided pleural fluid collection. At the level of the posterior ninth rib on the left, there was evidence of possible ICA injury (figure 2). Thoracic surgery was consulted and the decision was made to attempt embolisation of the lacerated ICA. However, due to the patient's diffuse atherosclerotic disease, vascular access was extremely difficult. During the procedure the patient went into cardiac arrest and died. At autopsy, the cause of death was hemothorax due to injury of the left ICA during thoracocentesis.

\section{DISCUSSION}

These three cases from three different hospitals highlight the possibility of ICA injury from routine pleural intervention, including one case using a $21 \mathrm{G}$ needle and two cases involving small gauge $8 \mathrm{~F}$ pigtail drains. One case was successfully managed with thoracotomy, one was successfully managed with angiographic embolisation of the bleeding ICA and another patient died during attempted embolisation.

Traditional anatomy teaching describes the ICA as lying in the intercostal groove, protected by the flange of the rib. This is the rationale behind the recommendation to insert needles just above the superior border of the rib. However, imaging studies have consistently demonstrated that the vessels lie exposed posteriorly, especially in elderly patients and in more cephalad rib

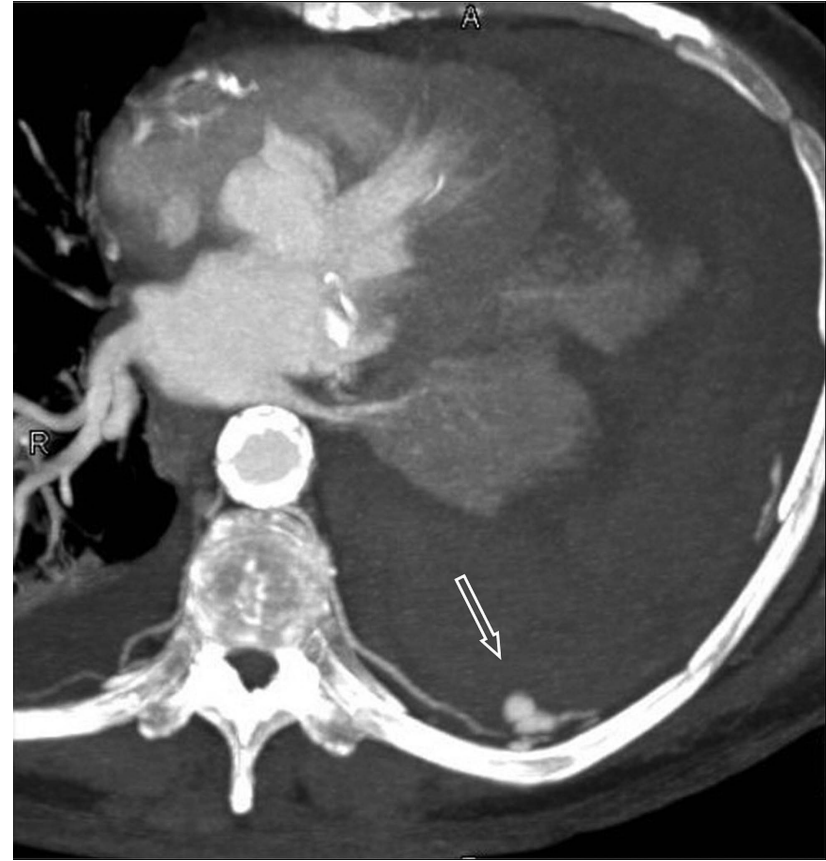

Figure 2 Case 3. CT angiogram shows the bleeding intercostal artery (open arrow).

spaces. ${ }^{3}$ For this reason, the posterior approach should be avoided if possible and a lateral approach, within the 'safe triangle', minimises risks of ICA laceration.

\section{Prevention of ICA laceration}

Current recommendations for chest drain insertion suggest that drains should be inserted in the 'safe triangle' in order to avoid the heart and the mediastinum and be above the level of the diaphragm. The safe triangle is formed anteriorly by the lateral border of the pectoralis major, laterally by the lateral border of the latismus dorsi, inferiorly by the line of the fifth intercostal space and superiorly by the base of the axilla ${ }^{1}$ (figure 3 ). Imaging guidance also aids in the safety of the procedure.

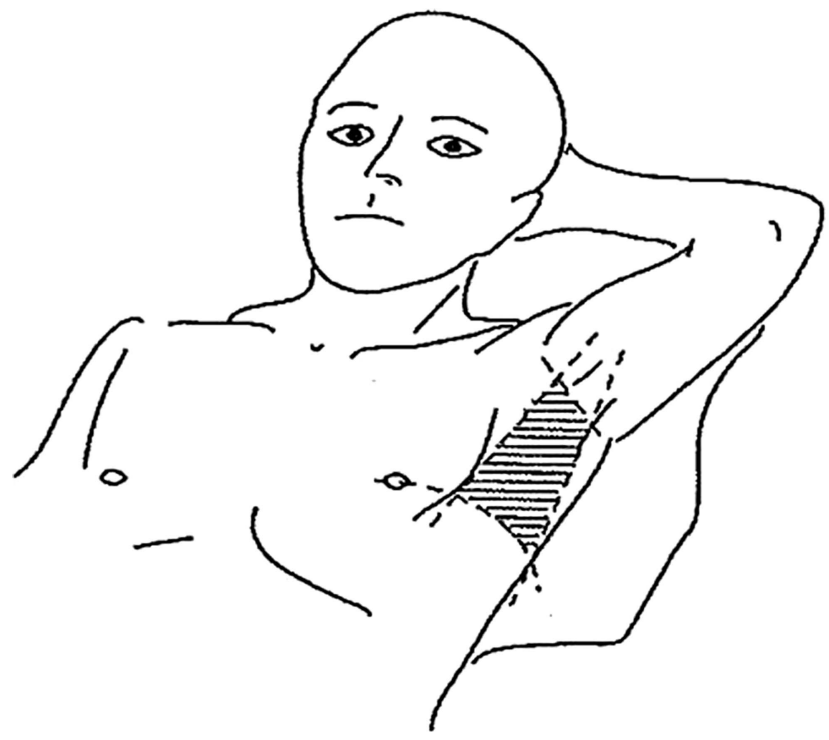

Figure 3 'Safe Triangle' (reproduced with permission from the British Thoracic Society guidelines on pleural procedures 2010). ${ }^{1}$ 
Salamonsen et $a l^{4}$ studied the use of thoracic ultrasound in determining the presence of the ICA within the intercostal space. Based on their findings, the identification of the ICA by using colour Doppler during thoracic ultrasound scanning may prevent laceration and it has been suggested as an important asset prior to pleural procedures.

\section{Recognition of ICA injury}

There are two main circumstances that can lead to potential fatality in ICA injury-either a failure to recognise the complication and/or a failure to rapidly institute appropriate and successful management. ICA injury may be obvious at the time of the procedure: for example, during thoracoscopy, on pleural fluid aspiration or during image-guided intervention. However, as illustrated in the above cases, the presentation may be delayed and injury may only be diagnosed when the patient becomes clinically unstable. It is therefore critical to have management protocols in place for the detection of haemorrhage. These include communication with the nursing staff that a procedure has been performed, regular post procedure observations and performing an early chest radiograph and repeat thoracic ultrasound. If there is a suspicion of ICA injury and the patient is sufficiently stable, ultrasound or CT of the thorax may be helpful in confirming the diagnosis.

\section{Management of ICA injury}

Standard resuscitative measures should be followed by specific measures to control the haemorrhage, particularly direct external pressure over the intercostal space through which the pleura was punctured. However, definitive management may require thoracic surgery or radiological intervention. Using angiographic techniques, it is possible to accurately localise the site of ICA haemorrhage and embolise the bleeding vessel. Chemelli et al reported their success rates in 24 cases of ICA injury, including 11 cases due to blunt trauma and 13 cases due to iatrogenic injury. Angiographic success was defined as successful ICA embolisation with complete occlusion of the bleeding source; they reported $91 \%$ success in traumatic injury and $85 \%$ success in iatrogenic injury.

\section{CONCLUSION}

This case series highlights the potential for ICA injury during apparently low-risk straightforward pleural procedures, the potentially serious morbidity and mortality of this complication and the importance of early diagnosis and intervention. All pleural units should have a standard operating procedure on the management of ICA injury to facilitate a rapid and appropriate response.

Contributors IP and NMR conceived the article, collected the data and wrote the manuscript. EJH, NAM, LY, DJF-K and FG provided data on cases and approved the final manuscript.

Competing interests None declared.

Provenance and peer review Not commissioned; externally peer reviewed.

\section{REFERENCES}

1 Havelock T, Teoh R, Laws D, et al. Pleural procedures and thoracic ultrasound: British Thoracic Society Pleural Disease Guideline 2010. Thorax 2010;65(Suppl 2):ii61-76.

2 Baldt MM, Bankier AA, Germann PS, et al. Complications after emergency tube thoracostomy: assessment with CT. Radiology 1995;195:539-43.

3 Helm EJ, Rahman NM, Talakoub 0, et al. Course and variation of the intercostal artery by CT scan. Chest 2013;143:634-9.

4 Salamonsen M, Dobeli K, McGrath D, et al. Physician-performed ultrasound can accurately screen for a vulnerable intercostal artery prior to chest drainage procedures. Respirology 2013;18:942-7.

5 Chemelli AP, Thauerer M, Wiedermann F, et al. Transcatheter arterial embolization for the management of iatrogenic and blunt traumatic intercostals artery injuries. J Vasc Surg 2009:49:1505-13. 